Classification

Physics Abstracts

$61.30-64.70 \mathrm{M}$

\title{
Cholesteric to cholesteric phase transitions in liquid crystals
}

\author{
H. R. Brand \\ ESPCI, 10, rue Vauquelin, F-75231 Paris Cedex 05, France \\ and H. Pleiner (*) \\ Physique des Solides, Bât. 510, Université Paris-Sud, F-91405 Orsay Cedex, France
}

(Reçu le 18 décembre 1984, révisé le 11 juin 1985, accepté le 11 juin 1985)

\begin{abstract}
Résumé. - Nous discutons les transitions qui pourraient apparaître entre deux phases cholestériques et nous expliquons par des arguments de symétrie générale pourquoi cette transition doit être toujours du premier ordre. Nous dérivons la dynamique macroscopique proche de la transition et nous proposons une expérience simple pour déplacer la température critique en utilisant un nouveau couplage réversible entre le paramètre d'ordre de la transition et des rotations inhomogènes.

Abstract. - We discuss the phase transitions which can occur between two different cholesteric phases and we present general symmetry arguments why this transition has to be always of the first order. We derive the macroscopic dynamics close to the phase transition and we propose an experiment to shift the critical temperature which makes use of a novel reversible cross coupling between the order parameter of the transition and inhomogeneous rotations.
\end{abstract}

In nematic liquid crystals [1] molecules align on average spontaneously along a certain direction, denoted by the so-called director $\hat{\mathbf{n}}$. In cholesteric liquid crystals [1] the molecules spiral in addition in a helical way around a preferred direction $\hat{\mathbf{p}}$, the pitch axis with a characteristic length scale, the pitch $2 \pi / q_{0}$. Locally these cholesteric liquid crystals are biaxial whereas globally, i.e. on length scales large compared to the pitch, cholesterics are uniaxial.

Very recently [2] it has become clear that biaxial nematic liquid crystals, which are characterized by nematic order in two perpendicular directions $\hat{\mathbf{n}}$ and $\mathbf{m}$ say (and thus in all three directions) and which have been studied extensively after their discovery [3] in lyotropic systems, both experimentally [4-9] and theoretically [10-16], also possess a cholesteric counter-part which has properties different from those of the usual cholesteric phase [17]. This novel type of cholesteric which we will call cholesteric II, is obtained when twist along one direction (say $\hat{\mathbf{n}} \times \hat{\mathbf{m}}$ ) is introduced in a biaxial nematic; i.e. $\hat{\mathbf{n}}$ and $\mathbf{m}$ spiral both around the preferred direction $\hat{\mathbf{n}} \times \mathbf{m}$ with one pitch $2 \pi / q_{0}$. As has been pointed out in reference [2] this phase has only defects with a singular core. We would like to stress that there are drastic quantitative differences in the degree of local biaxiality between cholesteric II and the usual cholesterics (hence-forth called cholesteric I). In cholesteric I the biaxial order is of a magnitude proportional to the square of the ratio of the molecular length to the pitch length (this is a number of the order of $10^{-8}$ in the lyotropic systems

$\left.{ }^{*}\right)$ Present and permanent address : Dept. Physics, Universität Essen, D43 Essen 1, F.R.G. 
we are focussing on in the present note predominantly) and in fact it appears that it has never been detected in a lyotropic system. In cholesteric II the order is locally isomorphic to that of a biaxial nematic and thus the degree of biaxial order must be comparable to those [3, 5]. This big change in local biaxiality immediately suggests an experimental tool to study the transition : NMR, which has proven to be so successful already in identifying $[3,5]$ the uniaxial to biaxial transition in nematics. This technique can be expected to be of comparable value for the cholesteric I to cholesteric II transition in large pitch systems since locally the similarity is striking.

As cholesterics I, cholesterics II are locally biaxial and globally uniaxial thus having the same symmetry properties in the ground state. From this fact it is immediately clear that the phase transition between cholesteric I and cholesteric II is of first order. The only other possibility being compatible with the general symmetry properties is that they could be identical which would mean no phase transition at all, i.e., a second order phase transition is clearly ruled out. This situation is reminiscent of the gas-liquid transition in simple fluids; the transition between the gas and the liquid phase is either first order or there is no difference at all (above the critical point which is the end of a line of first order phase transitions).

It is important to remember that the uniaxial to biaxial phase transition in nematics can be of second order (the symmetry changes from uniaxial to biaxial, globally) and this is found to be the case in all systems investigated experimentally [3-9]. Nevertheless the study of the behaviour of the cholesteric I-cholesteric II transition will be most interesting, in particular for mixtures (or pure compounds in a - so far hypothetical - thermotropic system) in which the transition is only weakly first order. This can be e.g. expected to be the case if one adds a sufficiently small amount of a chiral material to a lyotropic system (in this case the resulting pitch can be expected to be quite large - in analogy with results familiar from chiralizing thermotropic compounds showing uniaxial nematic phases) showing a second order uniaxial to biaxial nematic transition [23]. In figures 1 and 2 we have sketched the topology of several phase diagrams which might
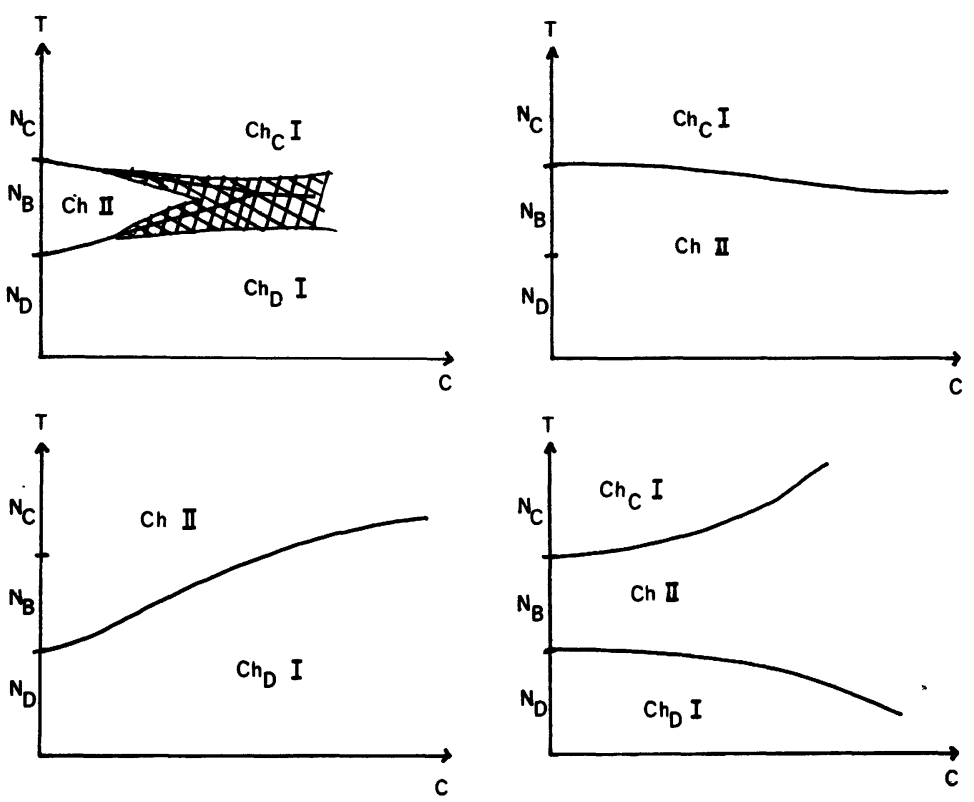

Fig. 1. - Phase diagrams which can arise when a lyotropic mixture which shows a biaxial and two different (rodlike and disklike) uniaxial nematic phases is chiralized; one example of a two phase region is shown (in cross-hatching). 
emerge by such a procedure. As one increases the amount of the chiral admixture we anticipate the cholesteric II to cholesteric I transition to become strongly first order. In figure 1 we have indicated one example of such a two phase region. As the cholesteric II to cholesteric I transition becomes more strongly first order, generally the temperature range of two phase regions will increase. This could give rise eventually to a complete suppression of the cholesteric II phase. Instead one obtains a two phase region associated with a strongly first order transition between a cholesteric I phase made of rodlike micelles and a cholesteric I phase made of disklike micelles (in a lyotropic system).

To derive the macroscopic dynamics close to the cholesteric I-cholesteric II phase transition we have to generalize the hydrodynamic description [18-20] valid in either phase [2]. In addition to the hydrodynamic variables we have to keep in our list of macroscopic quantities the variables which have - close to the transition cholesteric I-cholesteric II - a large, but finite relaxation time. In biaxial nematics one has the macroscopic order parameter [1]

$$
Q_{i j}=S\left(n_{i} n_{j}-\frac{1}{3} \delta_{i j}\right)+\tilde{T}\left(m_{i} m_{j}-(\mathbf{n} \times \mathbf{m})_{i}(\mathbf{n} \times \mathbf{m})_{j}\right)
$$

where the first term represents the uniaxial nematic order and the second is different from zero only in the biaxial nematic phase. Close to the transition the microscopic quantity $\tilde{T}$ becomes a macroscopic, slowly varying variable. It is the same quantity $\tilde{T}$ which becomes a slowly varying variable close to the cholesteric I-cholesteric II transition. In contrast to nematics, $\tilde{T}$ is nonzero, but microscopically small (of order $\left({ }^{1}\right) a^{2} q_{0}^{2}$ ) in cholesteric I whereas it is comparable in magnitude to the $\tilde{T}$ of biaxial nematics in cholesterics II. In cholesteric I, the nonzero, but microscopically small value of $\tilde{T}$ results from the induced biaxiality due to the helical structure and vanishes
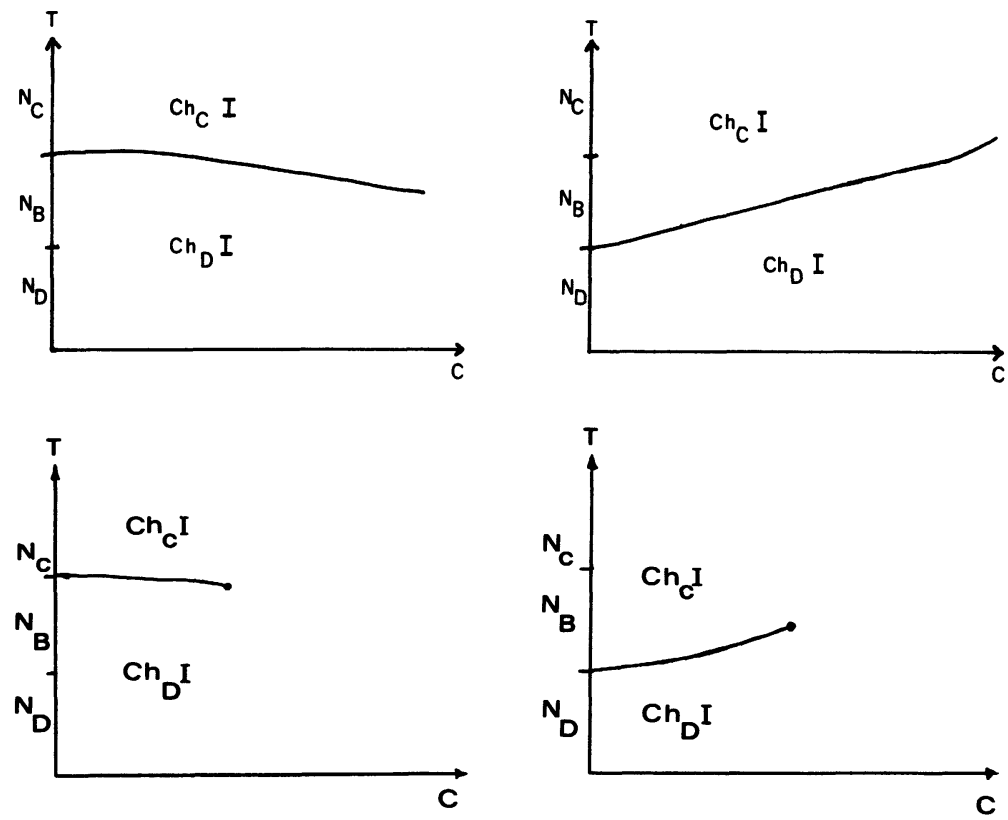

Fig. 2. - Same as figure 1 with cholesteric II being suppressed.

(1) With $a$ the molecular length. 
for $q_{0} \rightarrow 0$. In cholesteric II, $\tilde{T}$ describes the degree of order of the second molecular axis w.r.t. the second preferred axis $\hat{\mathbf{m}}$, as in biaxial nematics. In terms of Eulerian angles, $\tilde{T}$ can be defined [24] as

$$
\tilde{T}=\left\langle\frac{1}{4}\left(1+\cos ^{2} \Theta\right) \cos 2 \varphi \cos 2 \psi\right\rangle .
$$

If a Landau expansion is done for the whole $\tilde{T}$, we have

$$
F \sim \int \mathrm{d} \tau\left(\tilde{a} \tilde{T}+b \tilde{T}^{2}+c \tilde{T}^{3}+d \tilde{T}^{4}\right)
$$

with $\tilde{a} \sim q_{0}^{2}$, an expression which resembles that of a ferromagnet-paramagnet transition in an external field or of an imperfect bifurcation. Our statement that the cholesteric I to cholesteric II transition is first order applies if one uses the order parameter $\delta \tilde{T}$ with the value of $\tilde{T}$ in cholesteric I subtracted from the whole value. The jump of $\delta \tilde{T}$ at the transition is small because the value of $\tilde{T}$ in cholesteric $I$ is also small.

These fluctuations $\delta \tilde{T}$ seem to be the only natural candidate for a slow, i.e. macroscopic variable supplementing the usual truly hydrodynamic variables density $\rho$, entropy density $\sigma$, density of linear momentum $\mathbf{g}$ and displacement $u$ parallel to the pitch axis $\hat{\mathbf{p}}$ characteristic of the hydrodynamics of cholesteric liquid crystals [18-20] which is valid on length scales large compared to the pitch $[18,19]$.

For the static free energy we have

$$
F=F_{\text {Chol }}+\int \mathrm{d}^{3} r\left\{\alpha_{2}(\delta \rho)(\delta \tilde{T})+\alpha_{3}(\delta \sigma)(\delta \tilde{T})+\gamma_{3}(\delta \tilde{T})\left(\nabla_{3} u\right)+\frac{a}{2}(\delta \tilde{T})^{2}\right\}+F(\{\delta \tilde{T}\})
$$

where $F_{\text {Chol }}$ is the static free energy of a cholesteric [18, 19], $\gamma_{3}$ describes a change of the measured pitch due to an external variation of $\delta \tilde{T}$. The coefficients $\alpha_{2}, \alpha_{3}$ and $\gamma_{3}$ are proportional to $\langle\tilde{T}\rangle$ in Ginzburg-Landau approximation. The order parameter susceptibility is expected to show a large increase close to the transition, although $\chi_{\delta \tilde{T} \delta \tilde{T}}=\left[a^{\prime}\left(T-T^{*}\right)\right]^{-1}$ does not diverge.

Using the Gibbs relation

$$
T \mathrm{~d} \sigma=\mathrm{d} \varepsilon+\mu \mathrm{d} \rho+\mathbf{V} \cdot \mathrm{dg}+\phi_{i} \mathrm{~d} \nabla_{i} u+\psi \mathrm{d} \tilde{T}
$$

one can easily express the thermodynamic conjugate quantities $T, \mu, \mathbf{V}, \psi$ and $\phi_{i}$ in terms of the variables by taking variational derivatives of equation (2), e.g. $\phi_{i}=\left.\frac{\delta F}{\delta \nabla_{i} u}\right|_{\ldots}$.

The conservation laws

$$
\begin{array}{r}
\dot{\rho}+\operatorname{div} \mathbf{g}=0 \\
\dot{g}_{i}+\nabla_{j} \sigma_{i j}=0 \\
\dot{\sigma}+\nabla_{i} j_{i}^{\sigma}=0
\end{array}
$$

and the quasiconservation law for the variable characterizing the broken symmetry

$$
\dot{u}+X=0
$$

appropriate for the hydrodynamics of cholesteric liquid crystals has to be supplemented by the balance equation for fluctuations of the order parameter

$$
\dot{\tilde{T}}+Y=0 \text {. }
$$

To close the system of macroscopic equations we have to express the currents $\mathbf{g}, \sigma_{i j}, j_{i}^{\sigma}$ and $X$ as 
well as $Y$ in terms of the thermodynamic conjugates. For the irreversible currents which change compared to usual cholesterics [19] we have

$$
X^{\mathbf{D}}=\xi p_{j} \nabla_{j} T+\zeta \nabla_{j} \phi_{j}+\eta q_{0} \psi
$$

and

$$
Y^{\mathbf{D}}=\tau^{-1} \psi-\eta q_{0} \nabla_{j} \phi_{j}
$$

where $\zeta$ and $\xi$ are as in cholesterics [19]. In writing down equation (7) we have kept a term $\sim q_{0} \nabla_{j} \phi_{j}$ although it is of higher order in the wavevector, because the counter term in $X^{\mathrm{D}}$ (Eq. (6)) is $\sim q_{0} \psi$. The terms proportional to $\xi$ and $\eta$ reflect a dissipative coupling of temperature and order parameter to the effective pitch.

The term $\sim q_{0} \psi$ is allowed by symmetry in cholesterics due to the existence of the pseudoscalar $q_{0}$, a term absent in smectics A and discotic liquid crystals. A dissipative cross-coupling between order parameter variations and those of the layer displacement $u$ does not seem to have been considered before.

For the reversible currents we have

$$
\begin{aligned}
& \mathbf{g}^{\mathbf{R}}=\rho \mathbf{V} \\
& \sigma_{i j}^{\mathrm{R}}=\beta_{i j} \psi+\gamma q_{0}\left(\varepsilon_{m i k} p_{j} p_{m}+\varepsilon_{m j k} p_{i} p_{m}\right) \nabla_{k} \psi+p \delta_{i j}- \quad-p_{i} \phi_{j}-\left(\frac{1}{2 q_{0}} \varepsilon_{i j k}+q_{0} p_{k} g_{i j}\right) p_{k} \nabla_{l} \phi_{l} \\
& X^{\mathbf{R}}=V_{i} p_{i}-\frac{1}{2} p_{i} \varepsilon_{i j k} q_{0} \nabla_{j} V_{k}+g_{k l} \nabla_{k} V_{l} \\
& Y^{\mathbf{R}}=\beta_{i j} \nabla_{i} V_{j}+\gamma q_{0}\left(\varepsilon_{m i k} p_{j} p_{m}+\varepsilon_{m j k} p_{i l m}\right) \nabla_{i} \nabla_{j} V_{k} .
\end{aligned}
$$

Equations (8) are given in reference [19] with the terms $\sim g_{k l}$ added in reference [20]. In (9) the term $\sim \beta_{i j}$ is isomorphic to similar terms near the nematic-smectic $\mathrm{A}$ [21] and the nematicdiscotic [22] transition. This term gives rise to some degree of layer ordering if a flow with

$$
\beta_{\|} \nabla_{\|} V_{\|}+\beta_{\perp} \nabla_{\perp} V_{\perp} \neq 0
$$

is applied to a sample.

The second term in (9) (proportional to $\gamma$ ) is unique to systems which have a pseudoscalar quantity $q_{0}$, the pitch - a feature which is typical for systems with a helix. Thus such a contribution can only be expected close to a transition involving a helical liquid crystal phase. This term is susceptible to an experimental test close to a cholesteric I-cholesteric II transition. One prepares a layer of a material showing these two transitions in the planar configuration, i.e. with the helical axis perpendicular to the glass plates. Then one rotates the upper plate while keeping the lower one fixed (as sketched in Fig. 3), i.e. one applies a linearly inhomogeneous rotation. The amplitude of this rotation has to be chosen sufficiently small so as not to introduce too big distortions of the helical structure or instabilities. This seems feasible in the systems considered here due to the large pitch $(\sim 20 \mu \mathrm{m}$, i.e. already fairly close to a nematic). If the experiment is done in a lyotropic mixture which contains a small amount of chiral impurities the cholesteric Icholesteric II transition is nearly second order and thus the order parameter susceptibility $\chi_{\delta \tilde{T} \delta \tilde{T}}$ can be expected to become very large and enhance the degree of rotation-induced order. On a long time scale the amplitude will become large and defects will occur thus setting an upper bound for the time over which the effect predicted can be studied. (This situation is similar to that of the experiments performed by Janossy (J. Physique Lett. 42 (1981) 43) on the motion of impurities in cholesteric phases). 


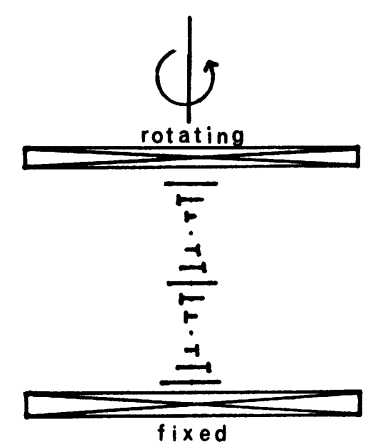

Fig. 3. - Configuration to change experimentally $T_{c}$ by applying an external inhomogeneous rotation.

We obtain for the value of $\tilde{T}$ via equations (7) and (9)

$$
\tau^{-1} a \delta \tilde{T}=\gamma q_{c}\left(\varepsilon_{m j k} p_{i} p_{m}+\varepsilon_{m i k} p_{j} p_{m}\right) \nabla_{i} \nabla_{j} V_{k}
$$

with $j, k$ in the $x y$-plane and $z$ parallel to the helical axis we have

$$
\delta \tilde{T}=q_{0} \gamma \frac{\tau}{a} \Lambda
$$

with $\Lambda \equiv \nabla_{z}\left(\nabla_{y} V_{x}-\nabla_{x} V_{y}\right)$

This induced finite value of $\tilde{T}$ can lead to a shift in the transition temperature for the cholesteric I-cholesteric II transition which one can detect.

If one is interested in a continuum-type description on length scales smaller than the pitch, one uses the nematic director $\hat{\mathbf{n}}$ as a variable (instead of $u$ ); the pitch $2 \pi / q_{0}$ is expressed by $\hat{\mathbf{n}}$ via $q_{0}=-\hat{\mathbf{n}} \cdot \operatorname{curl} \hat{\mathbf{n}}$. Here we focus on the changes when compared to the uniaxial-biaxial transition in nematics discussed in references [12] and [13].

For $\hat{\mathbf{n}}$ and $T$ we have the dynamic equations

$$
n_{i}+X_{i}=0 \text { and } \dot{\tilde{T}}+Y=0
$$

and for the reversible parts of $X_{i}$ and $Y$ we have

and

$$
X_{i}^{\mathrm{R}}=\left(\lambda_{i j k}+\frac{1}{2} q_{0} \varepsilon_{i j k}\right) \nabla_{j} V_{k}
$$

$$
Y^{\mathbf{R}}=\widetilde{\beta}_{i j} \nabla_{i} V_{j}+\tilde{\gamma} q_{0}\left(\varepsilon_{m i k} p_{j} p_{m}+\varepsilon_{m j k} p_{i} p_{m}\right) \nabla_{i} \nabla_{j} V_{k}
$$

where the expression (13) is as in uniaxial nematics [18] and where (14) is closely related to (9) given above; $\gamma \equiv \tilde{\gamma}$ and $\widetilde{\beta}_{i j}=\widetilde{\beta}_{1} \delta_{i j}+\widetilde{\beta}_{2} n_{i}^{0} n_{j}^{0}$.

Assuming for $\hat{\mathbf{n}}^{0}$ the configuration (helix axis parallel to $\hat{\mathbf{z}}$ )

$$
\hat{\mathbf{n}}^{0}=\hat{\mathbf{e}}_{x} \cos \varphi+\hat{\mathbf{e}}_{y} \sin \varphi
$$

and averaging yields

$$
\begin{aligned}
& \widetilde{\beta}_{x x}=\widetilde{\beta}_{1}+\frac{1}{2} \widetilde{\beta}_{2} \equiv \beta_{\perp} \\
& \widetilde{\beta}_{y y}=\widetilde{\beta}_{1}+\frac{1}{2} \widetilde{\beta}_{2} \equiv \beta_{\perp} \\
& \widetilde{\beta}_{z z}=\widetilde{\beta}_{1} \equiv \beta_{\perp},
\end{aligned}
$$


i.e. we see immediately that all the reversible contributions to $\dot{\tilde{T}}$ change continuously when going from a description which applies locally to one which is valid on length scales large compared to the pitch. This implies that it is irrelevant whether the experiment proposed in figure 3 is carried out for a sample thickness small or large compared to the pitch.

In conclusion we have discussed cholesteric-cholesteric transitions in liquid crystals and we have pointed out on the basis of general symmetry arguments that this transition has to be always first order. By chiralizing a material showing a second order uniaxial-biaxial nematic transition one might, however, be able to get a cholesteric I-cholesteric II transition which is only very weakly first order.

The derivation of the macroscopic dynamics can be used to test the influence of the helix close to a transition between two helical phases and a simple experiment involving an inhomogeneous rotation has been put forward. Experiments along these lines will certainly open new ways in our understanding of the role of chirality on a macroscopic scale.

\section{Acknowledgments.}

We would like to thank Yves Galerne, Lionel Liébert and Antonio M. F. Neto for discussing their recent experimental results with us. Support by the Deutsche Forschungsgemeinschaft is gratefully acknowledged.

\section{Note added :}

After this paper has been submitted for publication we received a preprint of reference [17] which reports three cholesteric phases in a lyotropic system obtained by chiralizing a mixture which has two different uniaxial and one biaxial nematic phase.

\section{References}

[1] Cf. e.g. De Gennes, P. G., The Physics of Liquid Crystals (Clarendon Press, Oxford) 1973.

[2] Pleiner, H. and Brand, H. R., Phys. Rev. Lett. 54 (1985) 1817 and submitted to Phys. Rev. A.

[3] Yu, L. J. and Saupe, A., Phys. Rev. Lett. 45 (1980) 1000.

[4] Bartolino, R., Chiaranza, T., Meuti, M. and Compagnoni, R., Phys. Rev. A 26 (1982) 1116.

[5] Saupe, A., Boonbrahm, P. and Yu, L. J., J. Chim. Phys. 80 (1983) 7.

[6] Meuti, M., Chiaranza, T. and Bartolino, R., Solid State Commun. 48 (1983) 751.

[7] Saupe, A., Nuovo Cimento D 3 (1984) 16.

Meuti, M., Barbero, G., Chiaranza, F. and Simoni, F., Nuovo Cimento D 3 (1984) 30.

[8] Galerne, Y. and Marcerou, J. P., Phys. Rev. Lett. 51 (1983) 2109.

[9] Roy, M., MCClymer, J. P. and KeYes, P. H., preprint Nov. 1984.

[10] Brand, H. and Pleiner, H., Phys. Rev. A 24 (1981) 2777.

[11] SAupe, A., J. Chem. Phys. 75 (1981) 5118.

[12] JACOBSEn, E. A. and Swift, J., Mol. Cryst. Liq. Cryst. 87 (1982) 29.

[13] Brand, H. and SwifT, J., J. Physique Lett. 44 (1983) L-333.

[14] Cajas, C. A., Swift, J. B. and Brand, H. R., Phys. Rev. A 28 (1983) 512.

[15] Cajas, C. A., Swift, J. B. and Brand, H. R., Phys. Rev. A 30 (1984) 1579.

[16] Pleiner, H. and Brand, H. R., J. Physique 46 (1985) 615.

[17] Neto, A. M. F., Galerne, Y. and LiéBeRT, L., preprint Feb. 1985, have recently observed such a transition.

[18] Martin, P. C., Parodi, O. and Pershan, P. S., Phys. Rev. A 6 (1972) 2401.

[19] Lubensky, T. C., Phys. Rev. A 6 (1972) 452.

[20] Brand, H. and Pleiner, H., J. Physique 41 (1980) 553. 
[21] LiU, M., Phys. Rev. A 19 (1979) 2090.

[22] BRAND, H. R., submitted for publication.

[23] This expectation turns out to be correct. The experiments carried out so far did not show any hysteresis (cf. Ref. [17]).

[24] Priest, R. G. and Lubensky, T. C., Phys. Rev. A 9 (1974) 893. 